interventions at appropriate stages in the lifecourse, we aim to provide robust and timely evidence which informs policies to improve population and individual health. A good example is provided by research into osteoporosis and age-related fractures. The Hertfordshire Cohort Study has established that birthweight and weight in infancy are important determinants of adult bone mass, microstructure, geometry and strength. These influence the later risk of fracture. Mother-offspring cohorts have demonstrated the key role played by maternal vitamin D insufficiency during pregnancy, in the intrauterine skeletal development of the offspring. These have led to formulation of intervention studies such as a randomised controlled trial of vitamin D supplementation during pregnancy.

\section{$02-2.5$ LIFESTYLE EXPOSURE MEASUREMENT IN COHORT STUDIES}

doi:10.1136/jech.2011.142976a.56

N Wareham. ${ }^{*}$ MRC Epidemiology Unit, Cambridge, UK

Previous cohort studies have demonstrated associations between lifestyle factors and chronic disease outcomes. However, for many of these factors such as physical activity, the exposure measurement has been relatively imprecise. The development of newer questionnaires that assess different domains of activity (transportation, work, domestic life and recreation) and inactivity (specific sedentary activities) may allow the associations between different aspects of these complex exposures and chronic disease to be disentangled. In general the precision of estimation is enhanced by shortening the time frame of reference. Therefore in order to optimise assessment of "usual" level of exposure it may be preferable to utilise repeated measurement with a questionnaire with a short time of reference rather than single use of one with a longer time frame. The increasing use of objective assessment of lifestyle factors alongside questionnaire based methods is an important step. Many questionnaires and objective instruments are available but need to be selected for a particular study on the basis of fitness for purpose. The
MRC Public Health Sciences Network has funded the development of a web-based toolkit describing these different approaches. This toolkit, which is available at http://www.dapa-toolkit.mrc.ac.uk/ will form the basis for future developments including enhancing wider access to computational software.

\section{2-2.6 REALISING THE POTENTIAL FOR INTERDISCIPLINARY PERSPECTIVES IN LIFE COURSE EPIDEMIOLOGY: A NEW BIRTH COHORT STUDY FOR THE UK}

doi:10.1136/jech.2011.142976a.57

C Dezateux. ${ }^{*}$ MRC Centre of Epidemiology for Child Health, London, UK

Introduction It is widely recognised that the UK birth cohorts have generated important new knowledge through life course analyses. However a new data resource is needed to advance understanding of the complex interplay between biology, behaviour, and environment during early development, and its influence on future health and well-being. An innovative design for a new UK Birth Cohort Study (BCS) was developed by a UK-wide investigator network in response to an open call for proposals. The preparatory phase will start in 2012

Methods The new UK BCS will recruit over 90000 pregnant mothers and their partners who will be seen during pregnancy and again, with their child, 4 months and 1 year after birth. Information, biological samples and biophysical assessments collected at these visits will be enhanced by linkage to obstetric records, environmental data collected from the home and a wide range of routine data sources. A further 20000 pregnant women throughout the UK will be interviewed when their children are aged 4 months and again at 1 year to allow the larger sample to be related to the UK population as a whole. In contrast to earlier UK-wide birth cohorts, the UK BCS will: collect data and biological samples from children and their families before birth and in early childhood; capture the substantial demographic changes that have taken place in the UK in recent years; and have sufficient power to identify effects specific for different ethnic groups. 